\title{
Design of measuring tool for the pitch of chain plate
}

\author{
Kang $\mathrm{Yu}^{1, \mathrm{a}}$, Jiguang $\mathrm{Han}^{2, \mathrm{~b} *}$ \\ ${ }^{1}$ School of Mechanical and Electrical engineering, Jiangsu Normal University, Xuzhou, China \\ ${ }^{2}$ School of Mechanical and Electrical engineering, Jiangsu Normal University, Xuzhou, China

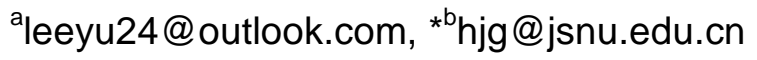

Keywords: chain plate; pitch; measuring tool

Abstract: As a significate part of chain, chain plate is not only connecting links but also under tension. The characters of dimension of chain plate were studied. It is obvious that the center distance of holes is very important. A study on measuring the center distance of holes of chains is conducted to meet the requirement of manufacturing and assembling. Research on the method of measuring center distance of holes is done. The measuring tool is designed according to direct measurement. The measuring head is designed on the basis of the principle of cone self-centering. The device employs grating for its measuring part. The method and the standard of how to adjust the measuring tool are presented in this paper.

\section{Introduction}

Chain drive, one of the widely used transmission form, transfers movement and power through the mesh of roller and sprocket. In comparison with gear trains, chain could be used in the situation of large center distance. It also works under the condition of high temperature, humidity, pollution and another harsh environment. Compared to belt, chain drive has the accurately average transmission ratio, no elastic sliding and slipping. Chain driver is widely used in all kinds of industrial products. With the rapid development of automobile industry, petroleum industry, agricultural machinery and the improvement of the degree of refinement of mechanical equipments, the market demand of the chain will be more and more. At the same time, the requirement of higher quality of the chain has been put forward.

Different versions of chains are used in different situations ${ }^{[1]}$. For instance, conveyor chain is used for conveying goods or production line. Agricultural chain is used for agricultural machinery, such as combine harvester, rice transplanter. The components of a chain of different types are investigated to obtain elemental components. A chain in mainly composed of inner plate, outer plate, pin and bush. Rollers are employed in roller chains but leaf chains have no roller. These components are connected in the form of hinge. Plate is an indispensable element of a chain. The quality of the plate affects the quality of the whole chain. The improvement of the quality of plate has great significance of the quality to a chain ${ }^{[2]}$.

\section{Analysis of Chain Plate's Dimension}

Chain plates have different shapes and sizes, but the elemental shape is similar. The shape could be simplified a plate with two holes. A mechanical drawing of chain plate is shown in Fig. 1. From Fig. 1 the highest accuracy requirement of the plate is the dimension of center distance between two holes, namely pitch. Conventional measuring instruments do not work efficiently in measuring the component with high precision mass production. Also precision of the conventional measuring instrument can't satisfy the demand of mass production. Special measuring tool would work efficiently in the situation. 


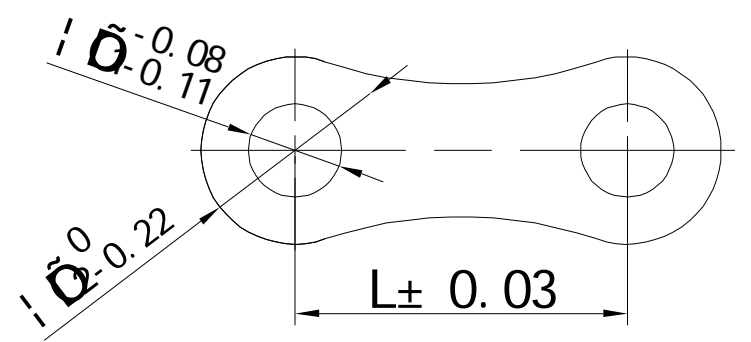

Fig. 1 Chain Plate

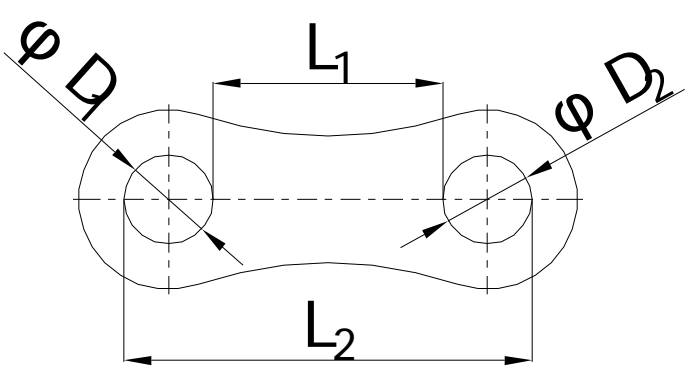

Fig. 2 Diagram of Indirect Method

\section{Method of Indirect Measuring Plate Pitch}

Indirect method: Fig. 2 shows that the inner diameters of two holes on plate were measured by vernier caliper respectively. The inner diameters were record as $D_{1}$ and $D_{2}$. $D_{1}$ equals to $D_{2}$ or not. Then we measured the minimum and maximum distances between the two holes. Record the related distance as $\mathrm{L}_{1}$ and $\mathrm{L}_{2}$. The pitch can be easily determined by solving Eq. 1, Eq. 2 and Eq. 3. The value of $\mathrm{L}$ is the pitch of plate.

$$
\begin{aligned}
& \mathrm{L}^{\prime}=\mathrm{L}_{1}+\left(\mathrm{D}_{1}+\mathrm{D}_{2}\right) / 2 . \\
& \mathrm{L}^{\prime \prime}=\mathrm{L}_{2}-\left(\mathrm{D}_{1}+\mathrm{D}_{2}\right) / 2 . \\
& \mathrm{L}=\left(\mathrm{L}^{\prime}+\mathrm{L}^{\prime \prime}\right) / 2 .
\end{aligned}
$$

Measurement method: The method of measuring pitch in factory is shown in Fig. 3. A couple of chain plates were assembled with two mandrels. The mandrels were manufactured according to the different diameters of plate's hole. The mandrels and plate hole should match firmly. Vernier caliper is employed to measure the minimum and maximum distance between two mandrels. Therefore the values of $L_{1}$ and $L_{2}$ are obtained. $D_{1}$ and $D_{2}$ are represented by $D_{0} . D_{0}$ is a constant diameter of mandrels so that it is convenient for calculating pitch. The value of $\mathrm{D}_{0}$ should smaller than $\mathrm{L}$. The tolerances of manufacture were shown in Fig. 3.

$$
\begin{aligned}
& \mathrm{D}_{1 \min }=\mathrm{D}_{1}-0.11 . \\
& \mathrm{D}_{3}=\mathrm{D}_{1 \min }-0.1 .
\end{aligned}
$$

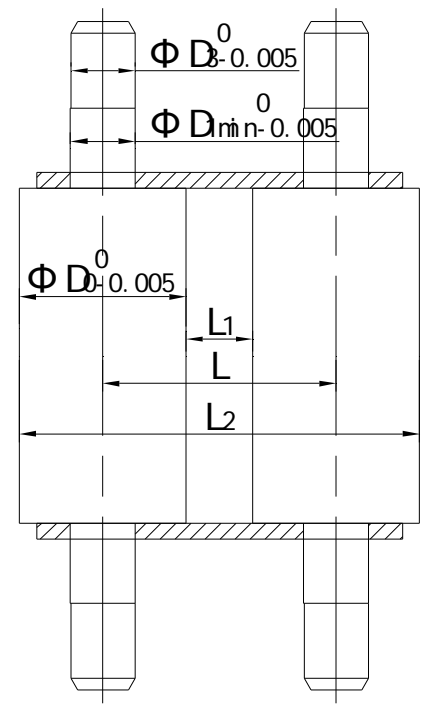

Fig. 3 Diagram of Mandrel Measurement of Pitch

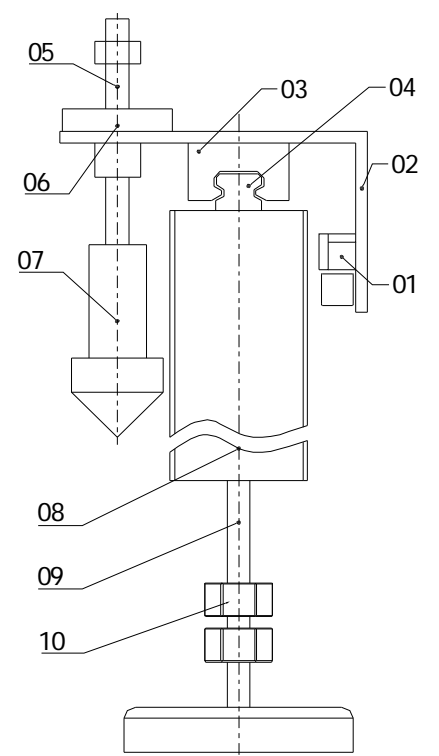

Fig. 4 Assembly Drawing of Measuring Tool 


\section{Method of Direct Measuring Plate Pitch}

First a reference hole should be set before measuring the pitch. Then measure the distance from the reference hole to other hole. The distance is plate's pitch which we wanted. The measuring head should have the function of self centering. The center line of a cone could coincide with center line of hole. Cone head could be well positioned in the center of a hole. The measuring head was designed on the basis of cone. The pitch is obtained by measuring the distance of movement of the cone head. The measurement error caused by manufacturing tolerance could be avoided by using the same cone head as measuring head.

\section{Structure Design of Measuring Tool}

Structure of measuring tool as shown in Fig. 4, including the main support part, measuring part (grating ruler and digital display table), the side of the head part (locate head and so on).

01Grating ruler $(500 \mathrm{~mm}$ ) 02Support plate 03Slider 04Guide rail 05Joint lever 06Location bush 07Locate head 08Support frame 09Support leg 10Nut

The support part of the measuring instrument is made of $60 \times 60$ standard hard aluminum material, which can be used to adjust the height and the level of the measuring tool. According to the requirements of the measuring tools design, the accuracy of the general measuring tools is a magnitude higher than the parts ${ }^{[3]}$, such as distance of two hole centers $L \pm 0.03$, vernier caliper cannot reach this accuracy, but grating ruler can meet the requirements, and can measure different length of center distance, it is unlike fixed length gauge only measure the same size specifications.

\section{Design of Measuring Head}

As shown in Fig. 4, the head part ${ }^{[4]}$ is composed of a connecting rod, a positioning sleeve and a locate head. The specific design plan as follows.

The locate head is made of wear-resisting, high hardness materials, such as Cr12 manufacturing, and after heat treatment, the hardness reached HRC58-62, roughness 0.4, as is shown in Fig. 5 can be used to measure the locate head diameter 0 chain plate hole heart to 40 hole distance, and distance measuring hole mandrel method greatly increased compared with general gage. In the process of measuring, the position head needs to move up and down and ensure the verticality, the end of the locate head is designed in accordance with the principle of design for the hammer Morse of 1:20 hole, combined with the connecting rod in Fig. 6, the divided design of the connecting rod and the locate head design as the following two reasons: the first reason is to consider the exchange for different locate head or other measuring heads; and the second reason is to consider to increase the utilization rate of materials, and if you choose the first plan and you need to use bars at least diameter 45 , and it will increase the cutting time and waste the materials.

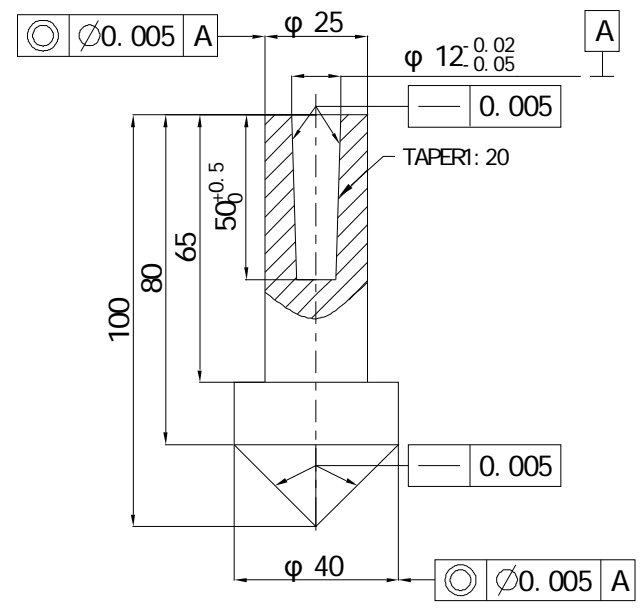

Fig. 5 Drawing of Locate Head

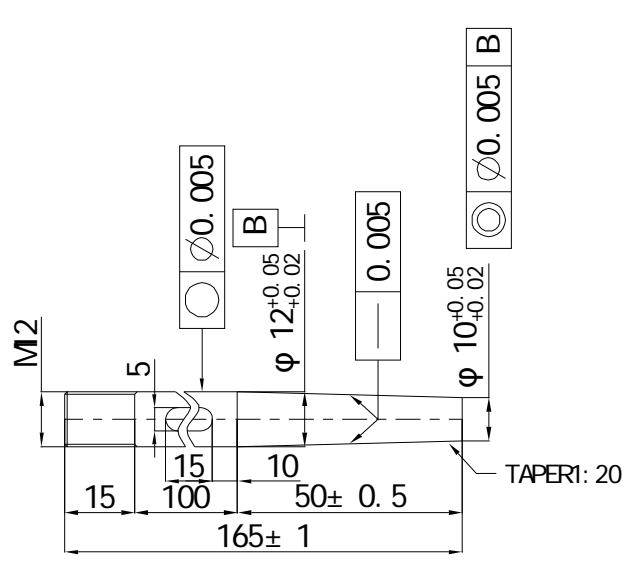

Fig. 6 Drawing of Joint Lever 
The drawing of location bush is shown in Fig. 7. The location bush fixed on the support plate. The measuring head connected the joint lever firmly. The joint lever could slide up and down in the hole of location bush so that the measuring head could move up and down to adapt the various diameters of holes of plate. The joint lever should well match up with the location bush. The tolerance range of joint lever is from 0 to $+0.01 \mathrm{~mm}$. The tolerance range of the hole in location bush is from $-0.01 \mathrm{~mm}$ to 0 . Both matching surface roughness are $0.4^{[5]}$. A dial indicator was employed to ensure the tolerance of fit while installation. Hardness and good abrasion resistance material, such as $\mathrm{Cr} 12, \mathrm{Cr} 14$, was chosen for manufacturing this part. Then this part's hardness reached HRC 58-62 after heat treatment. The lower surface of the location bush needs to close contact with the support plate surface. The groove ensures the perpendicularity of measuring head. The flatness of surface of location bush is 0.005 and surface roughness is 0.4 . The better surface finish is provided by grinding.

A spring could be added to end of the joint lever after the installation of the entire measuring head. The measuring head gained the function of automatic reset because of the elastic of the spring. The spring was not drawn in picture. The rest roughness of contact surfaces in the Figures is 0.4 through polishing and grinding.

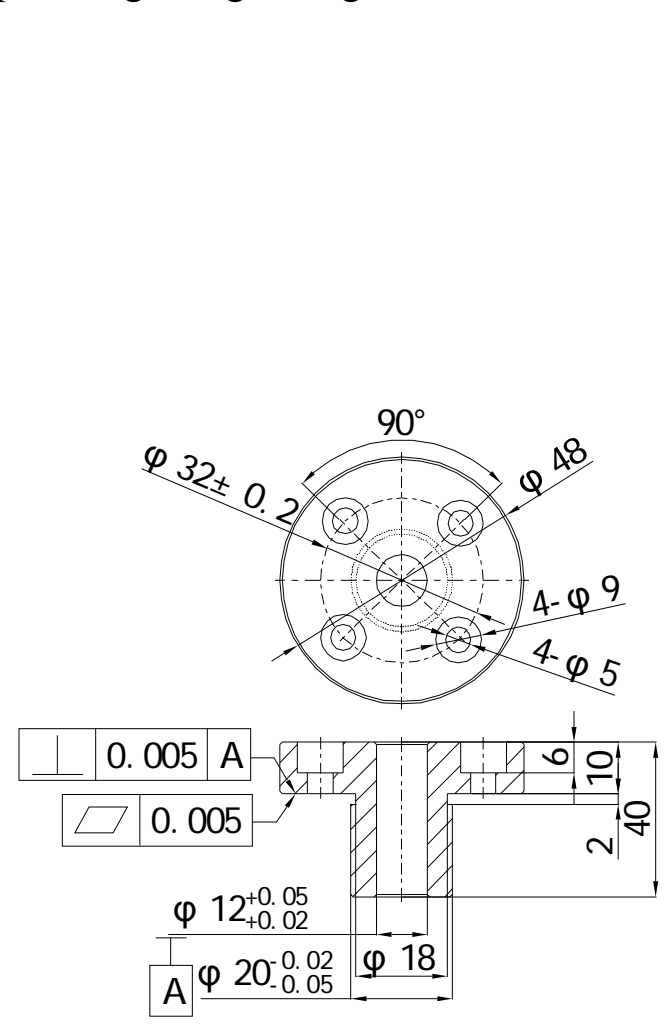

Fig. 7 Drawing of Locate Bush
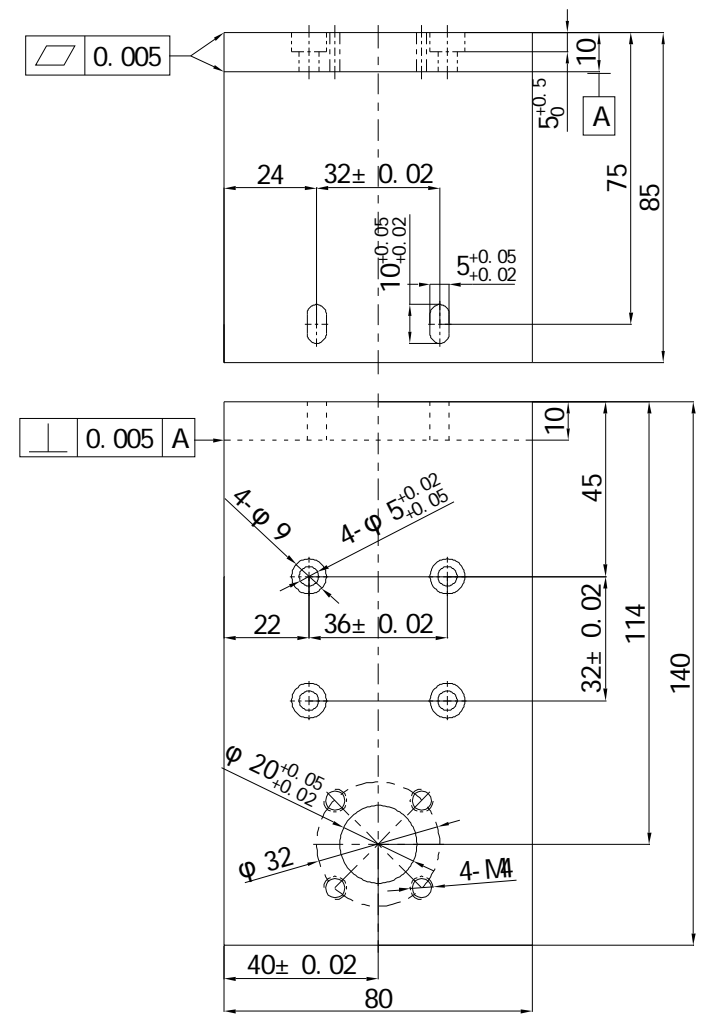

Fig. 8 Drawing of Support Plate

\section{Connection of parts}

Move the measuring head when measuring pitch of a plate. At the same time, the measuring head drives the grating ruler. The sensor of the grating ruler gained the signal and conveyed it to the digital display table. The measuring results are obtained. The part of measurement, part of measuring head and support part are assembled together with slider and guide rail via support plate(as shown in Fig. 8). The material of the support plate should have sufficient strength and stiffness to resist deformation. Type 45 steel was chosen for support plate. Smooth the surface and ensure the flatness of 0.005 . According to the types of slider and guide rail supplied by companies, the slider and guider rail of type BGXH20BN of SFAF satisfied the requirement of measuring tool. The instruction should be followed to install a slider and guide rail. The slider and guide rail should be well oiled.

\section{Calibration of the Measuring Tool}

The measuring tool should be assembled according to the requirements of assembling. Calibration of the measuring tool should be given before use. Firstly, the installation between components should 
be fastened. Secondly, the slider or moving parts should be flexible and well oiled. Thirdly, the transmission of signal between grating ruler and digital display table should be normal. Fourthly, measure the pitch of sets of plates and record the results. The quantity of measurement is 10 times at least. Its poor value of $1 / 10-1 / 14$ of pitch tolerance could be accepted ${ }^{[6]}$. Fifth, use a three-coordinates measuring machine to calibrate the measuring tool.

\section{Conclusions}

The current measuring mandrel for measuring the pitch is relatively complicated. It could not work efficiently. The versatility of the current measuring mandrels is not strong. Different measuring mandrels were manufactured for different chain plate. It is also not convenient at the situation of large or small pitch. The pitch can't be obtained directly.

In this paper a new measuring tool is designed. The versatility of the new measuring tool is strong though the cost is higher. Different pitch can be measured by the same measuring head conveniently. The results display directly on the digital display table. Different sizes of measuring heads meet the requirements of different occasions by replacing measuring head. This measuring tool also can be used to measure linear dimension of other components. This new measuring tool has a certain practical significance.

\section{References}

[1] Weiyang Zuo, Jiguang Han, Machine Design and Manufacturing Engineering, 44 (2015) 73-75. In Chinese

[2] Qingfa Ji, Journal of Jilin Institute of Technology, 00 (1980) 90-101. In Chinese

[3] Longhai Chen, Manufacture Information Engineering of China, 39 (2010) 64-67. In Chinese

[4] Lin Zhou, Jing Zhou, Yanqing Yang, Mechanical Engineer, 11 (2013) 154-155. In Chinese

[5] Bangchun Wen, Machinery's Handbook, fifth ed., China Machine Press, Beijing, 2010.

[6] Xiaojiang Yuan, Machine Design and Research, 25 (2009) 75-76, 95. In Chinese 